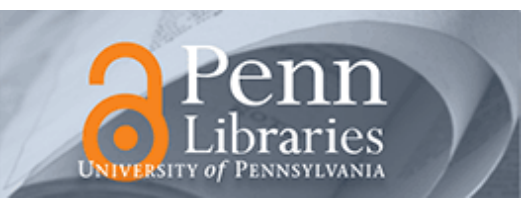

University of Pennsylvania

ScholarlyCommons

$10-31-2010$

\title{
Optimal Robust Multihop Routing for Wireless Networks of Mobile Micro Autonomous Systems
}

Jonathan Fink

University of Pennsylvania, jonfınk@seas.upenn.edu

Alejandro Ribeiro

University of Pennsylvania, aribeiro@seas.upenn.edu

Vijay Kumar

University of Pennsylvania, kumar@grasp.upenn.edu

Brian M. Sadler

Army Research Laboratory

Follow this and additional works at: https://repository.upenn.edu/ese_papers

Part of the Electrical and Computer Engineering Commons

\section{Recommended Citation}

Jonathan Fink, Alejandro Ribeiro, Vijay Kumar, and Brian M. Sadler, "Optimal Robust Multihop Routing for Wireless Networks of Mobile Micro Autonomous Systems", . October 2010.

Suggested Citation:

J. Fink, A. Ribeiro, V. Kumar and B.M. Sadler. (2010). "Optimal Robust Multihop Routing for Wireless Networks of Mobile Micro Autonomous Systems." The 2010 Military Communications Conference. San Jose, CA. October 31 November 3, 2010.

C2010 IEEE. Personal use of this material is permitted. However, permission to reprint/republish this material for advertising or promotional purposes or for creating new collective works for resale or redistribution to servers or lists, or to reuse any copyrighted component of this work in other works must be obtained from the IEEE.

This paper is posted at ScholarlyCommons. https://repository.upenn.edu/ese_papers/588

For more information, please contact repository@pobox.upenn.edu. 


\title{
Optimal Robust Multihop Routing for Wireless Networks of Mobile Micro Autonomous Systems
}

\author{
Abstract \\ This paper develops algorithms to ensure that agents of a mobile micro autonomous system (MMAS) \\ maintain integrity of communication flows as they move to accomplish their task. Due to inherent \\ uncertainties in estimation of wireless channels, we advocate a stochastic approach whereby achievable \\ communication rates of point-to-point links are regarded as random variables with known means and \\ variances. To achieve reliable end-to-end communication flows, terminals route their traffic through \\ various alternative paths to reduce the effect of uncertainty in individual link rates. The proposed \\ algorithms are optimal and robust in that routes are obtained as solutions of optimization problems \\ subject to constraints on minimum required rates and maximum acceptable variances. Algorithms are \\ tested in an event-based simulator that uses an accurate data-driven model of radio communications to \\ model both the structure of code running independently on multiple robots as well as the transmission of \\ messages via a real radio. Simulation results corroborate that rates of end-to-end flows are maintained at \\ target levels despite variations in the rates of individual links.

\section{Disciplines} \\ Electrical and Computer Engineering | Engineering

\section{Comments} \\ Suggested Citation: \\ J. Fink, A. Ribeiro, V. Kumar and B.M. Sadler. (2010). "Optimal Robust Multihop Routing for Wireless \\ Networks of Mobile Micro Autonomous Systems." The 2010 Military Communications Conference. San \\ Jose, CA. October 31 - November 3, 2010. \\ (C2010 IEEE. Personal use of this material is permitted. However, permission to reprint/republish this \\ material for advertising or promotional purposes or for creating new collective works for resale or \\ redistribution to servers or lists, or to reuse any copyrighted component of this work in other works must \\ be obtained from the IEEE.
}




\section{Optimal Robust Multihop Routing for Wireless Networks of Mobile Micro Autonomous Systems}

\author{
Jonathan Fink, Alejandro Ribeiro, Vijay Kumar \\ University of Pennsylvania
}

\begin{abstract}
This paper develops algorithms to ensure that agents of a mobile micro autonomous system (MMAS) maintain integrity of communication flows as they move to accomplish their task. Due to inherent uncertainties in estimation of wireless channels, we advocate a stochastic approach whereby achievable communication rates of point-to-point links are regarded as random variables with known means and variances. To achieve reliable end-to-end communication flows, terminals route their traffic through various alternative paths to reduce the effect of uncertainty in individual link rates. The proposed algorithms are optimal and robust in that routes are obtained as solutions of optimization problems subject to constraints on minimum required rates and maximum acceptable variances. Algorithms are tested in an event-based simulator that uses an accurate data-driven model of radio communications to model both the structure of code running independently on multiple robots as well as the transmission of messages via a real radio. Simulation results corroborate that rates of end-to-end flows are maintained at target levels despite variations in the rates of individual links.
\end{abstract}

\section{INTRODUCTION}

Mobile micro autonomous systems (MMAS) are composed of micro robots that are deployed as a team to accomplish a given task. To coordinate their actions, agents of the MMAS maintain reliable communication flows between each other and, possibly, with a central command center. Even though maintaining integrity of the communication flows may not be a goal per se, it is nonetheless a prerequisite for task accomplishment. Therefore, it is necessary to devise mechanisms to prompt MMAS agents to self configure a multihop wireless network to route information of prescribed communication flows. Since wireless links between agents change as they move, a more accurate statement is that it is necessary to design joint routing and mobility control algorithms that guarantee integrity of communication flows.

The problem of controlling mobility while ensuring integrity of the communication network dates back to the work on connectivity preserving rendezvous [1]. Over time, this has become an important problem in robotics with an extensive literature devoted to the preservation of links, the increase of network connectivity metrics, and recovery from disconnected topologies [2]-[10]. All of these works rely on disc models that identify the existence of a link with some form of physical proximity and define connectivity in terms of topological properties of the resulting graph.

Although the use of disc models and topological connectivity is consistent with early approaches to wireless networking [11], [12], it has long being recognized that identifying proximity with reliable communication is somewhat arbitrary, because even small differences in locations might result in dramatic differences in signal strength due to small scale fading [13], [14].
A more accurate model is to consider a link rate function that maps pairs of terminal positions to achievable communication rates. These functions can be as simple as measuring packet error probabilities [15], [16] or may involve more complex models accounting for fading and power adaptation [17], [18]. Either way, supporting communication flows becomes not a question of maintaining a connected graph, but of determining routing variables compatible with achievable point-to-point rates that yield target end-to-end communication rates. This is the approach advocated in this paper.

In the context of MMAS, the foremost challenge in determining routing variables is the variability of wireless channels and the consequent difficulty of predicting signal strength and communication rates, see e.g., Section IV-A. Indeed, since agents are moving, there is no time to estimate channels accurately as is done for terminals in fixed positions. Furthermore, channels at future positions cannot be measured and have to be estimated based on propagation models. Given this limitation it is natural to model link rates as random variables and design routing and mobility control algorithms based on statistical characterizations, e.g., means and variances, of link capacities.

An important observation is that if point-to-point link capacities become random, so do the rates of end-to-end communication flows. The design goal then becomes one of ensuring performance of end-to-end communication flows in a probabilistic sense leading to robust routing formulations [19]. In robust routing, the goal is to exploit spatial diversity by restricting robot's movements so that they stay within possible communication range of various peers. The uncertainty in end-to-end transmission rates is thus reduced as the deviations of pointto-point capacities from their estimates in different individual links tend to cancel each other. Robust routing is formulated here through the solution of pertinent optimization problems; see Section II. In these problems, target values in end-to-end average rates and variances are imposed as constraints. Since this still leaves leeway in the selection of routing variables, two separate optimality criteria are considered: maximization of average rate and minimization of variance.

Optimal routing variables are then used as inputs to mobility control algorithms that balance movement along a task gradient with the preservation of communication rates; see Section III. Specifically, we use means and variances of individual links to determine the probability that actual end-to-end rates stay above prescribed minimum required levels. Movements of agents are then constrained to regions that ensure that actual rates stay above minimum rates with high probability. The resultant algorithms are then tested in an event-based simulator that uses an accurate data-driven model of radio communications 
to model both the structure of code running independently on multiple robots as well as the transmission of messages via a real radio; see Section IV. We conclude the paper in Section V.

\section{OPTIMAL ROBUST ROUTING}

Consider a MMAS composed of $J$ agents, let $\left\{\mathbf{x}_{i}\right\}_{i=1}^{J}$ denote their corresponding positions, and denote as $\mathbf{x}:=$ $\left[\mathbf{x}_{1}^{T}, \ldots, \mathbf{x}_{J}^{T}\right]^{T}$ a vector grouping all positions. With agents $i$ and $j$ at positions $\mathbf{x}_{i}$ and $\mathbf{x}_{j}$ it is possible to deliver information from $i$ to $j$ at a rate of $R\left(\mathbf{x}_{i}, \mathbf{x}_{j}\right)$ units of information per units of time. The function $R\left(\mathbf{x}_{i}, \mathbf{x}_{j}\right)$ depends on, e.g., distances and the interference environment, but due to significant uncertainties inherent to wireless propagation it is not possible to predict $R\left(\mathbf{x}_{i}, \mathbf{x}_{j}\right)$ accurately. We therefore resort to a statistical characterization whereby $R\left(\mathbf{x}_{i}, \mathbf{x}_{j}\right)$ is regarded as a random quantity with known mean $\bar{R}\left(\mathbf{x}_{i}, \mathbf{x}_{j}\right)$ and variance $\tilde{R}\left(\mathbf{x}_{i}, \mathbf{x}_{j}\right)$. For some pairs of terminals $(i, j)$ we may have $\bar{R}\left(\mathbf{x}_{i}, \mathbf{x}_{j}\right)=0$ indicating that we expect that direct communication between $i$ and $j$ is not possible. Define then the neighborhood of $i$ as the set $n(i):=\left\{j: \bar{R}\left(\mathbf{x}_{i}, \mathbf{x}_{j}\right) \neq 0\right\}$ containing indices of terminals $j$ with which $i$ can communicate directly.

In order to accomplish their task, agents have to support $K$ information flows generically indexed by $k$. The set of destinations of the k-th information flow is denoted as $\operatorname{dest}(k)$ and we let $a_{i}^{k}$ for all $i \notin \operatorname{dest}(k)$ denote the rate at which terminal $i$ generates packets belonging to the $k$-th flow. Communication integrity requires information generation rates $a_{i}^{k}$ to exceed prescribed values.

In order to deliver information from generating sources $i$ to intended destinations in $\operatorname{dest}(k)$, agents rely on stochastic multihop communication through neighboring terminals. In every time slot, agent $i$ chooses to serve the flow $k$ by sending packets to the next hop $j$, with probability $\alpha_{i j}^{k}$. Considering that when $j$ is chosen as the next destination, $R\left(\mathbf{x}_{i}, \mathbf{x}_{j}\right)$ units of information are transmitted, the average rate at which $k$-flow packets leave terminal $i$ is $\sum_{j \in n(i)} \alpha_{i j}^{k} R\left(\mathbf{x}_{i}, \mathbf{x}_{j}\right)$. Similarly, the average rate at which $k$-flow packets are received at terminal $i$ is $\sum_{j \in n(i)} \alpha_{j i}^{k} R\left(\mathbf{x}_{j}, \mathbf{x}_{i}\right)$. The difference between the outgoing rate and the incoming rate determines the amount of information $a_{i}^{k}$ at which $i$ can accept packets for the $k$-th flow. Therefore, we have that for all $i \notin \operatorname{dest}(k)$ it must be

$$
a_{i}^{k}(\boldsymbol{\alpha}, \mathbf{x})=\sum_{j \in n(i)} \alpha_{i j}^{k} R\left(\mathbf{x}_{i}, \mathbf{x}_{j}\right)-\alpha_{j i}^{k} R\left(\mathbf{x}_{j}, \mathbf{x}_{i}\right),
$$

where for future reference we define the vector $\alpha$ grouping all routing variables $\alpha_{i j}^{k}$. Notice that in (1) it holds that $\alpha_{j i}^{k}=0$ for $j \in \operatorname{dest}(k)$, since destinations do not transmit their own packets. We must also have $\sum_{j \in n(i), k} \alpha_{i j}^{k} \leq 1$ since the $\alpha_{i j}^{k}$ are a set of probabilities at terminal $i$. Probabilities $\alpha_{i j}^{k}$ effectively determine the routing of information through the MMAS. Our goal is to determine routing variables $\alpha_{i j}^{k}$ to support prescribed rates.

If $R\left(\mathbf{x}_{i}, \mathbf{x}_{j}\right)$ is random, it follows from (1) that available communication rates $a_{i}^{k}$ are also random. Therefore, the goal of determining routing variables $\alpha_{i j}^{k}$ to support prescribed rates is expressed in a stochastic sense. For doing so, note that the expected value of $a_{i}^{k}(\boldsymbol{\alpha}, \mathbf{x})$ is

$$
\bar{a}_{i}^{k}(\boldsymbol{\alpha}, \mathbf{x}):=\mathbb{E}\left(a_{i}^{k}(\boldsymbol{\alpha}, \mathbf{x})\right)=\sum_{j \in n(i)} \alpha_{i j}^{k} \bar{R}\left(\mathbf{x}_{i}, \mathbf{x}_{j}\right)-\alpha_{j i}^{k} \bar{R}\left(\mathbf{x}_{j}, \mathbf{x}_{i}\right),
$$

while the variance of $a_{i}^{k}(\boldsymbol{\alpha}, \mathbf{x})$ is

$$
\tilde{a}_{i}^{k}(\boldsymbol{\alpha}, \mathbf{x}):=\operatorname{var}\left(a_{i}^{k}(\boldsymbol{\alpha}, \mathbf{x})\right)=\sum_{j \in n(i)} \alpha_{i j}^{k} \tilde{R}\left(\mathbf{x}_{i}, \mathbf{x}_{j}\right)+\alpha_{j i}^{k} \tilde{R}\left(\mathbf{x}_{j}, \mathbf{x}_{i}\right) .
$$

The interpretation of (2) and (3) is that variables $\alpha_{i j}^{k}$ control the probability distribution of achievable rates $a_{i}^{k}(\boldsymbol{\alpha}, \mathbf{x})$. We can thus define target average rates $\bar{a}_{0}$ and variances $\tilde{a}_{0}$ and state the problem of ensuring communication integrity as the determination of routing variables $\alpha_{i j}^{k}$ that yield average rates $\bar{a}_{i}^{k}(\boldsymbol{\alpha}, \mathbf{x}) \geq \bar{a}_{0}$ and variances $\tilde{a}_{i}^{k}(\boldsymbol{\alpha}, \mathbf{x}) \leq \tilde{a}_{0}$ that respectively exceed and do not exceed their corresponding targets.

Achieving target rates and variances still leaves leeway in the determination of $\alpha_{i j}^{k}$. This motivates the introduction of optimality criteria and corresponding optimization problems to select a particular set of $\alpha_{i j}^{k}$ among all those that satisfy the stated constraints. Introduce then a utility function $U\left(\bar{a}_{i}^{k}(\boldsymbol{\alpha}, \mathbf{x})\right)$ to measure the value of average rate and consider the optimization problem that maximizes the social value $\sum_{i, k} U\left(\bar{a}_{i}^{k}(\boldsymbol{\alpha}, \mathbf{x})\right)$ subject to achieving target rates and variances, i.e.,

$$
\begin{aligned}
& \boldsymbol{\alpha}(\mathbf{x})=\operatorname{argmax} \sum_{i, k} U\left(\bar{a}_{i}^{k}(\boldsymbol{\alpha}, \mathbf{x})\right) \\
& \text { s.t. } \quad \bar{a}_{0} \leq \bar{a}_{i}^{k}(\boldsymbol{\alpha}, \mathbf{x}) \leq \sum_{j \in n(i)} \alpha_{i j}^{k} \bar{R}\left(\mathbf{x}_{i}, \mathbf{x}_{j}\right)-\alpha_{j i}^{k} \bar{R}\left(\mathbf{x}_{j}, \mathbf{x}_{i}\right) \\
& \tilde{a}_{0} \geq \sum_{j \in n(i)} \alpha_{i j}^{k} \tilde{R}\left(\mathbf{x}_{i}, \mathbf{x}_{j}\right)+\alpha_{j i}^{k}{ }^{2} \tilde{R}\left(\mathbf{x}_{j}, \mathbf{x}_{i}\right) \\
& \sum_{j \in n(i), k} \alpha_{i j}^{k} \leq 1, \quad \alpha_{i j}^{k} \geq 0 .
\end{aligned}
$$

In (4) we use the mean and variance expressions in (2) and (3) and we also state explicitly the constraints on the probabilities $\alpha_{i j}^{k}$.

Alternatively, we can introduce a cost function $V\left(\tilde{a}_{i}^{k}(\boldsymbol{\alpha}, \mathbf{x})\right)$ associated with variance $\tilde{a}_{i}^{k}(\boldsymbol{\alpha}, \mathbf{x})$ and minimize the global cost $\sum_{i, k} V\left(\tilde{a}_{i}^{k}(\boldsymbol{\alpha}, \mathbf{x})\right)$, i.e,

$$
\begin{array}{ll}
\boldsymbol{\alpha}(\mathbf{x})= & \operatorname{argmin} \\
\text { s.t. } & \left.\tilde{a}_{0} \geq \tilde{a}_{i}^{k}(\boldsymbol{\alpha}, \mathbf{x}) \geq \sum_{j \in n(i)} \alpha_{i j}^{k}{ }^{2} \tilde{R}(\mathbf{\alpha}, \mathbf{x})\right) \\
& \bar{a}_{0} \leq \sum_{j \in n(i)} \alpha_{i j}^{k} \bar{R}\left(\mathbf{x}_{i}, \mathbf{x}_{j}\right)-\alpha_{j i}^{k}{ }^{2} \tilde{R}\left(\mathbf{x}_{j}, \mathbf{x}_{i}\right) \\
& \left.\sum_{j \in n(i), k} \alpha_{i j}^{k} \leq 1, \quad \mathbf{x}_{j}, \mathbf{x}_{i}\right)
\end{array}
$$

Analogously to (4), the minimization in (5) is with respect to routing variables that achieve target rates and variances.

Variables $\alpha_{i j}^{k}$ that solve (4) and (5) achieve target rates and variances. In (4) the indeterminacy for choice of $\alpha_{i j}^{k}$ is resolved by prioritizing large expected rates. In (5) the degrees 


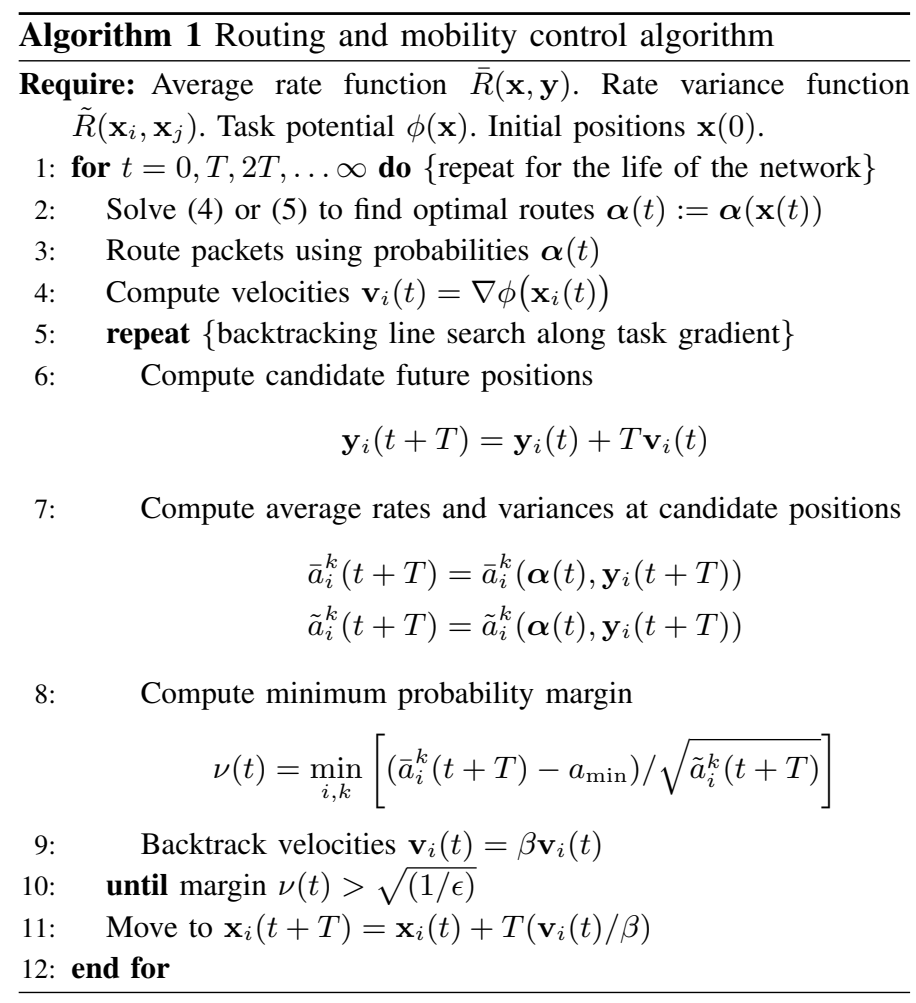

of freedom are used to prioritize small variability around target rates.

\section{ROUTING AND MOBILITY CONTROL}

The MMAS objective is determined by a task potential $\phi\left(\mathbf{x}_{i}\right)$, whose gradient $\nabla \phi\left(\mathbf{x}_{i}\right)$ determines the agents' movement. To be more specific introduce a time step $T$ and let $\mathbf{x}_{i}(t)$ denote the position of agent $i$ at time $t=k T$. The candidate velocity of agent $i$ is then set to $\mathbf{v}_{i}(t)=\nabla \phi\left(\mathbf{x}_{i}(t)\right)$ so that the candidate position $\mathbf{y}_{i}(t+T)$ of $i$ at time $t+T$ is

$$
\mathbf{y}_{i}(t+T)=\mathbf{x}_{i}(t)+T \mathbf{v}_{i}(t)
$$

As agents move, however, they have to maintain individual communication links capable of supporting required flow rates. Assume then that optimal routing variables $\boldsymbol{\alpha}(t):=\boldsymbol{\alpha}(\mathbf{x}(t))$ have been computed for current positions $\mathbf{x}(t)$ and define a minimum acceptable rate $a_{\min } \leq a_{0}$. If agents move to candidate positions $\mathbf{y}_{i}(t+T)$ as per (6), end-to-end rates $a_{i}^{k}(\boldsymbol{\alpha}(t), \mathbf{y}(t+T))$ are determined by (1). For the MMAS to be able to service $k$ th flow traffic originating at agent $i, a_{i}^{k}(\boldsymbol{\alpha}(t), \mathbf{y}(t+T))$ must exceed $a_{\text {min }}$, i.e., we must have $a_{i}^{k}(\boldsymbol{\alpha}(t), \mathbf{y}(t+T))>a_{\min }$. Recalling that rates $a_{i}^{k}(\boldsymbol{\alpha}(t), \mathbf{y}(t+T))$ in (1) are random, we further define a small target probability $\epsilon$ and require $a_{i}^{k} \geq a_{\min }$ with probability at least $1-\epsilon$. Equivalently

$$
\operatorname{Pr}\left[a_{i}^{k}(\boldsymbol{\alpha}(t), \mathbf{y}(t+T)) \leq a_{\min }\right]<\epsilon
$$

To compute the probabilities in (7) it is necessary to make a hypothesis on the probability distribution of $a_{i}^{k}(\boldsymbol{\alpha}(t), \mathbf{y}(t+$ $T)$ ), which in turns depends on the distribution of $R\left(\mathbf{y}_{i}(t+\right.$ $\left.T), \mathbf{y}_{j}(t+T)\right)$ and $R\left(\mathbf{y}_{j}(t+T), \mathbf{y}_{i}(t+T)\right)$ for all neighbors $j \in$ $n(i)$. Without making any assumptions, however, it is possible to bound the probabilities in (7) using Chebyshev's inequality to obtain an expression in terms of means $\bar{a}_{i}^{k}(\boldsymbol{\alpha}(t), \mathbf{y}(t+T))$ and variances $\tilde{a}_{i}^{k}(\boldsymbol{\alpha}(t), \mathbf{y}(t+T))$ as determined by (2) and (3) respectively. To simplify notation, define means and variances at target positions as

$$
\begin{aligned}
& \bar{a}_{i}^{k}(t+T)=\bar{a}_{i}^{k}(\boldsymbol{\alpha}(t), \mathbf{y}(t+T)) \\
& \tilde{a}_{i}^{k}(t+T)=\tilde{a}_{i}^{k}(\boldsymbol{\alpha}(t), \mathbf{y}(t+T)) .
\end{aligned}
$$

From Chebyshev's inequality we then conclude that a sufficient condition for (7) to be true is

$$
\nu_{i}^{k}(t):=\frac{\bar{a}_{i}^{k}(t+T)-a_{\min }}{\sqrt{\tilde{a}_{i}^{k}(t+T)}} \geq \sqrt{\frac{1}{\epsilon}},
$$

where we defined the probability margin $\nu_{i}^{k}(t)$.

Using specific assumptions on the distribution of $a_{i}^{k}(\boldsymbol{\alpha}(t), \mathbf{y}(t+T))$ tighter bounds can be obtained. If, e.g., we assume that $a_{i}^{k}(\boldsymbol{\alpha}(t), \mathbf{y}(t+T))$ has a Gaussian distribution, (7) is equivalent to

$$
\nu_{i}^{k}(t):=\frac{\bar{a}_{i}^{k}(t+T)-a_{\mathrm{min}}}{\sqrt{\tilde{a}_{i}^{k}(t+T)}} \geq C^{-1}(1-\epsilon),
$$

where $C^{-1}(x)$ is the inverse of the standard Gaussian cumulative distribution function. The expression in (10) and the bound in (9) have different right hand sides, but both of them are completely determined by the probability margin $\nu_{i}^{k}(t)$.

In order to ensure that communications of all flows $k$ and nodes $i$ are serviced by the MMAS the margins $\nu_{i}^{k}(t)$ have to satisfy (9) (or (10) if a Gaussian model is used). Since this need not be satisfied by candidate positions $\mathbf{y}_{i}(t+T)$ computed from (6) we perform a backtracking search on the vector $\mathbf{v}(t)$. Specifically, we define the minimum probability margin $\nu(t)$ as

$$
\nu(t):=\min _{i, k} \nu_{i}^{k}(t)=\min _{i, k}\left[\frac{\bar{a}_{i}^{k}(t+T)-a_{\min }}{\sqrt{\tilde{a}_{i}^{k}(t+T)}}\right] .
$$

If the minimum probability margin $\nu(t) \geq \sqrt{1 / \epsilon}$ we move with velocity $\mathbf{v}_{i}(t)$ to candidate position $\mathbf{y}_{i}(t+1)$. If $\nu(t)<\sqrt{1 / \epsilon}$ we scale the velocity to $\mathbf{v}_{i}(t)=\beta \mathbf{v}_{i}(t)$ using some backtracking parameter $\beta \in(0,1)$ and restart the process with the candidate position associated with the scaled velocity. The process is repeated until $\nu(t) \geq \sqrt{1 / \epsilon}$.

The routing and mobility control algorithm is summarized in Algorithm 1. The inputs to the algorithm are the mean rate function $\bar{R}(\mathbf{x}, \mathbf{y})$, the rate variance function $\tilde{R}\left(\mathbf{x}_{i}, \mathbf{x}_{j}\right)$, the task potential $\phi(\mathbf{x})$, and initial positions $\mathbf{x}(0)$. Parameters $\epsilon$, $\beta, a_{\min }, \bar{a}_{0}$, and $\tilde{a}_{0}$ also need to be specified. At any point in time, optimal routes $\boldsymbol{\alpha}(t):=\boldsymbol{\alpha}(\mathbf{x}(t))$ are computed by solving either (4) or (5) (Step 2). Routing variables $\boldsymbol{\alpha}(t)$ are used to route packets between times $t$ and $t+T$ (Step 3). The velocity vector is then computed using the task gradient (Step 4) before proceeding to a backtracking search with the objective of getting all constraints in (9) to be satisfied (steps 5-10). By ensuring that (9) is satisfied for all $i, k$, the backtracking search ensures satisfaction of (7) for all $i, k$ and consequently that all nodes and flows are serviced by the MMAS with high 

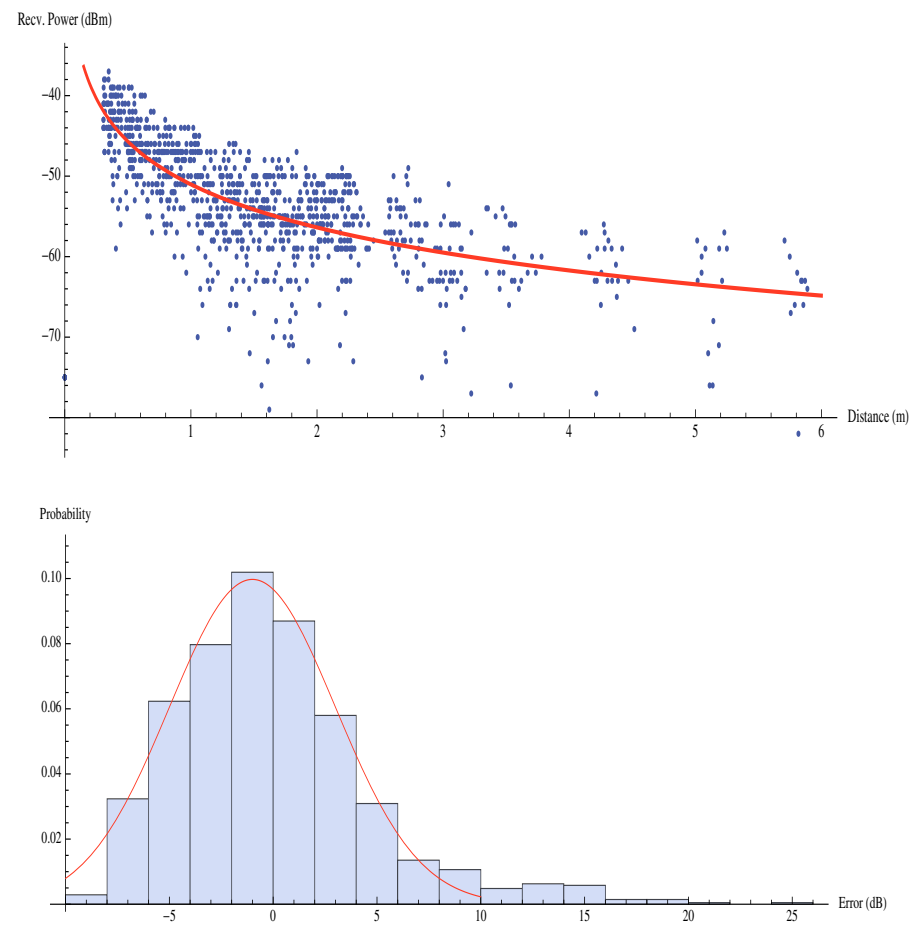

Fig. 1. Log-fading model fit to experimental data. Note that deviations from this mean function can be modeled by a Rician fading model or approximated by a non-zero-mean Gaussian

probability. The backtracking search starts with the computation of candidate positions $\mathbf{y}_{i}(t+T)$ (Step 6). Rate expected values $\bar{a}_{i}^{k}(t+T)$ and variances $\tilde{a}_{i}^{k}(t+T)$ at the target position $\mathbf{y}_{i}(t+T)$ using current optimal routes $\boldsymbol{\alpha}(t)$ are then found (Step 7). The minimum probability margin $\nu(t)$ is then computed (Step 8) and the velocity $\mathbf{v}(t)$ is backtracked according to the parameter $\beta$ (Step 9). Upon exiting the backtracking search, the velocity has been scaled to a value compatible with integrity of endto-end communication flows. The terminal then moves with velocity $\mathbf{v}_{i}(t) / \beta$ to position $\mathbf{x}_{i}(t+T)=\mathbf{x}_{i}(t)+T\left(\mathbf{v}_{i}(t) / \beta\right)$. The algorithm then proceeds to the re-computation of optimal routes by solving either (4) or (5) (Step 2).

\section{RESULTS}

Our end goal is to deploy these algorithms experimentally on a team of 10 to 15 small ground robots. Here we develop the software architecture and methods but keep the implementation in the realm of an event-based simulator that uses an accurate data-driven model of radio communications to model both the structure of code running independently on multiple robots as well as the transmission of messages via a real radio.

As an implementation detail, note that the optimization problem in (4) and (5) is a quadratic program (QP) and can be solved efficiently with the open-source library CGAL [20]. It is also true that this problem can be solved with a distributed sub-gradient descent algorithm without any centralization of information.

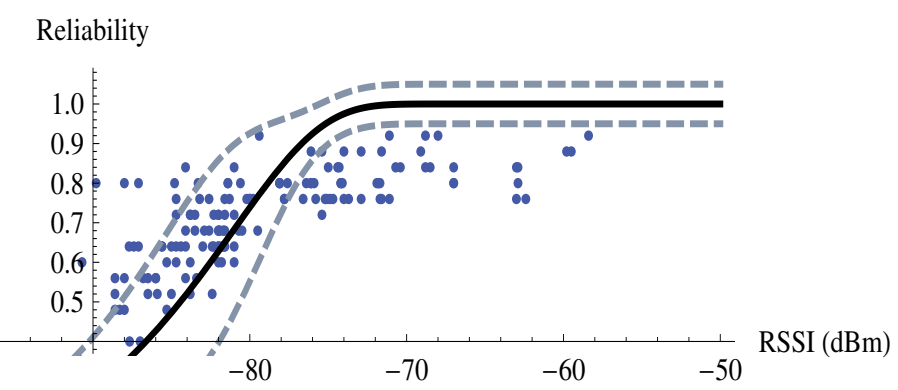

Fig. 2. Here we depict the received signal strength to link reliability mapping. Note that the dashed lines represent $\pm \sigma$ of the received signal strength. Even taking this into account, the experimental data is over estimated by the model (most likely due to non constant noise levels in the environment).

\section{A. Communication Models}

The robust approaches to wireless networking and mobility presented above are motivated by communication models that are stochastic in nature. We are interested in implementation of these methods on small, inexpensive, low power radio devices such as Zigbee (implementing the IEEE 802.15.4 standard). As a metric for current communication capability, these devices supply the user with received signal strength. Thus we will determine models necessary to map received signal strength to communication reliability or rate.

1) Radio Signal Propagation: Radio propagation is a complex multi-scale process. Received power is a function of distance from the source, shadowing due to obstacles, and multipath phenomena that arise as a result of reflections and refractions. While spatially and temporally averaged behavior can be fit to deterministic fading models, small-scale fading can cause variations to received signal strength on the order of $\pm 5 \mathrm{dBm}$ over small length scales. Though small-scale fading can be modeled by complex ray-tracing methods, it is perhaps more readily represented probabilistically by a Rician (when there $i s$ line-of-sight) or Rayleigh (for non-line-of-sight) distributions. Thus, the received power (in $\mathrm{dBm}$ ) can be given by

$$
P_{\mathrm{dBm}}=\underbrace{L_{0}-10 n \cdot \log \left(\left\|x^{s}-x\right\|\right)}_{\text {Fading }}-\underbrace{f\left(x^{s}, x\right)}_{\text {Shadowing }}-\underbrace{\epsilon}_{\text {Multipath }}
$$

where $L_{0}$ is the measured power at $1 \mathrm{~m}$ from the source, $n$ is the decay exponent, and $x^{s}, x$ are the positions of the source and receiver respectively, $f(\cdot, \cdot)$ is a non-smooth function that describes shadowing, and $\epsilon$ is drawn from a Rayleigh or Rician distribution.

Though (as shown in our previous work [14] and depicted in Fig. 1) a dense sampling of a particular environment can yield accurate parameter estimation, we wish to deploy our methods in unknown environments that can not be sampled a priori.

2) Communication Rate: Directly from [21], bit error rate $\left(p_{b}\right)$ and received power level $P_{r}$ are a function of the modulation scheme but can be generically related by

$$
p_{b} \propto \operatorname{erfc}\left(\sqrt{\frac{\text { constant } \times P_{r}}{N \times f}}\right)
$$




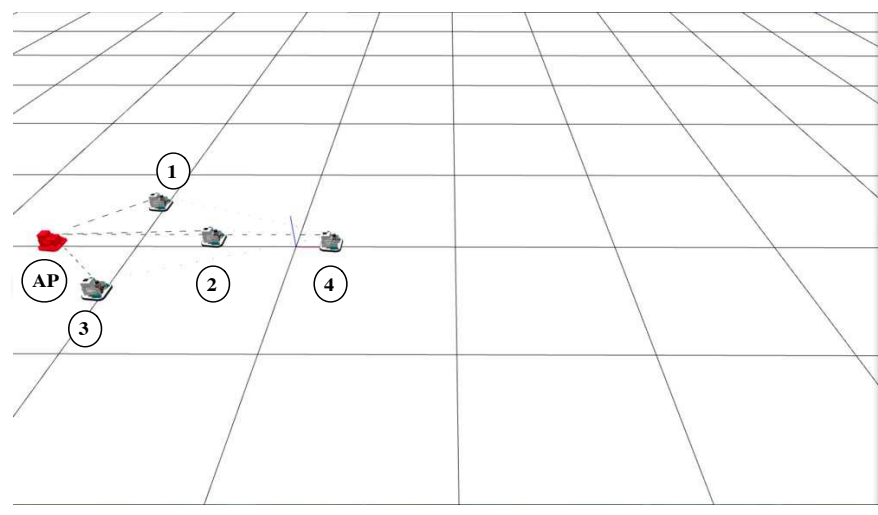

(a)

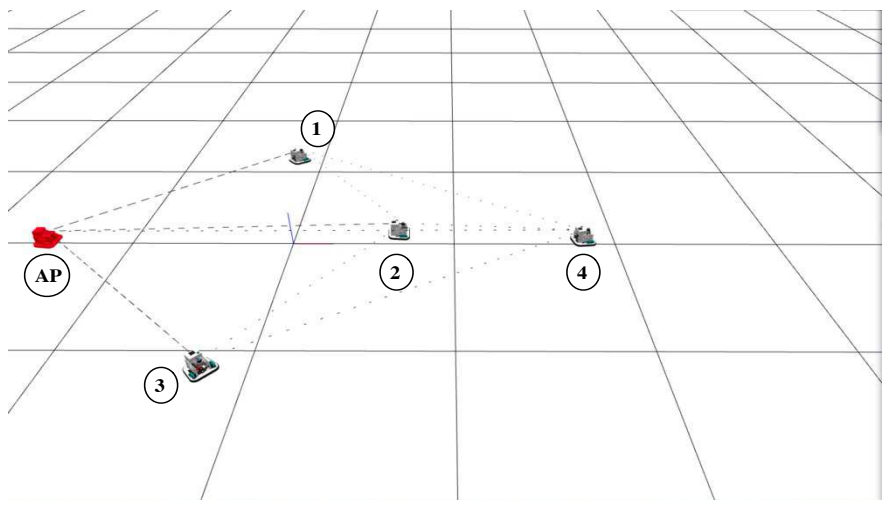

(c)

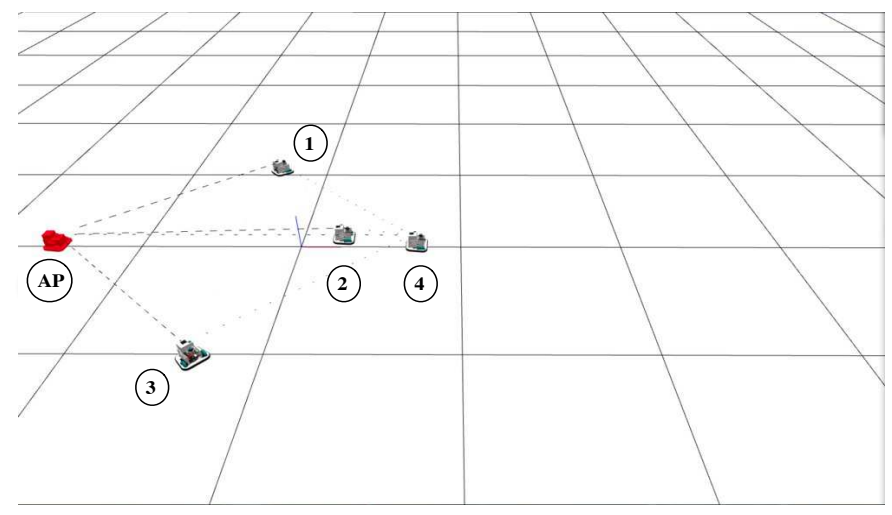

(b)

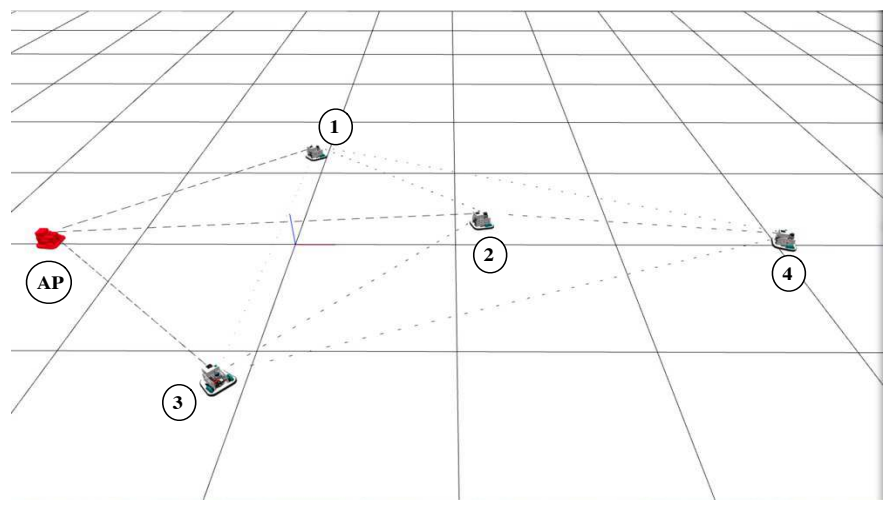

(d)

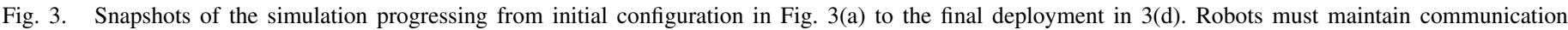

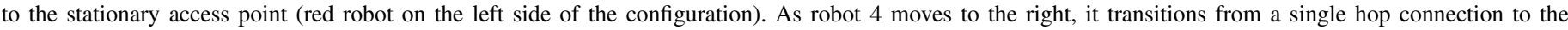

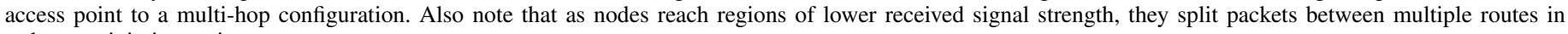
order to minimize variance.

where $N$ is the noise spectral density (noise power per hertz), $f$ is the raw channel bit rate, and $\operatorname{erfc}(x)$ is the complementary error function. Zigbee (802.15.4) employs a modulation scheme: O-QPSK with half-sine pulse shaping (MSK) with 16 channels spaced at $5 \mathrm{Mhz}$ and has transmission rates of $250 \mathrm{~kb} / \mathrm{s}$ [22]. Based on our experimental results, we use

$$
p_{b}=\operatorname{erfc} \sqrt{\frac{P_{r}}{N}}
$$

(with $N=-80 \mathrm{dBm}$ for our environment) to approximately relate the received signal strength with packet error rate.

The stochastic model of received signal strength can be applied to the communication rate mapping by a Taylor series expansion

$$
\operatorname{var}\left[p_{b}\left(P_{r}\right)\right] \approx\left(p_{b}^{\prime}\left(\mathbb{E}\left[P_{r}\right]\right)\right)^{2} \operatorname{var}\left[P_{r}\right] .
$$

With an additional uniform variance applied to account for a non-constant noise level, the mapping from received signal strength to link reliability is depicted in Fig. 2.

\section{B. Simulation Environment}

In order to facilitate a realistic simulation that can be easily transitioned to an experimental system, we employ a hardware abstraction layer and robotics operating system (ROS) [23]. At it's most simple, ROS provides a messaging system between independent pieces of software (whether algorithmic or a hardware driver). For example, we are able to construct a radio communications simulator that relies on (12), (14), and (15) while presenting exactly the same interface that a real Zigbee radio driver would provide.

In this way, we strove to follow the same methods we would in an experimental implementation. For instance, the current link estimates $\bar{R}, \tilde{R}$ in step 2 of Alg. 1 are determined by probing the network and computing statistics on packet arrivals over a short time window ( $2 \mathrm{~s}$ for these results) while the link predictions in step 7 of Alg. 1 are computed based on the models in (12)-(15). This type of methodology will enable a quick transition to ground robots with low-power radio devices.

\section{Coverage problem}

In this work, we define the coverage problem to be a velocity controller that seeks to disperse robots into the environment. Individual robot velocities are chosen to drive away from their neighbors. In this case, we are defining neighbors as robots with whom we can currently communicate.

While dispersing, we seek to maintain desired end-to-end rates $a_{\min }=0.2$ from each robot back to the access point. Figure 3 depicts a simulation trial of the coverage problem.

In this example, the initial configuration leads most robots to maintain single-hop connections to the access point and their 


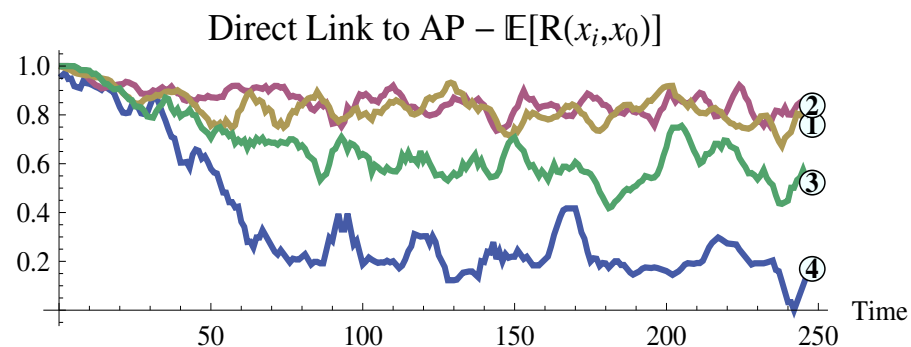

Fig. 4. Clearly, as the nodes disperse they move farther from the access point, resuling in decreased link capacity to the access point. Specifically, note that robot 4's link drops below the point of being able to support the desired rate causing it to route packets through its neighbors.

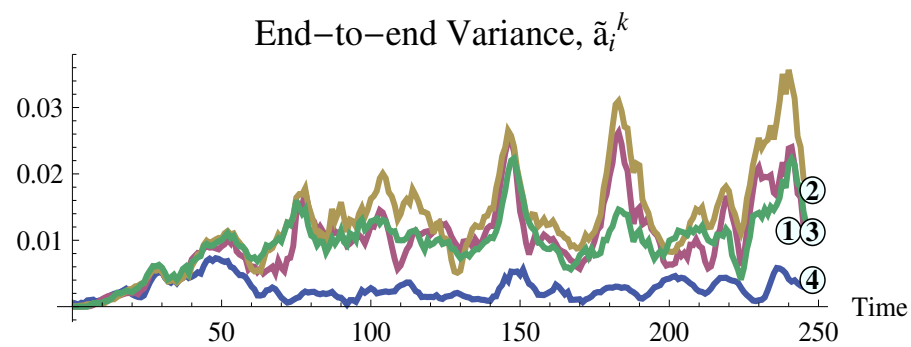

Fig. 5. Variance on the end-to-end rate of each robot back to the access point. While our routing solution is seeking to minimize the sum of end-to-end variances, robot 4 acheives the lowest variance when it stops sending packets directly to the access point and instead splits them between neighbors.

motions are limited primarily by the capacity of that single link. However, the robot on the far right in Fig. 3(d) moves such that its minimum rate cannot be maintained by a direct link to the access point (as depicted in Fig. 4) and it splits its packets between neighbors so they are then routed back to the access point. This splitting actually serves to decrease the variance on this particular end-to-end rate as shown in Fig. 5.

\section{CONCLUSions}

Robust algorithms to ensure integrity of end-to-end communication flows in mobile micro autonomous systems (MMAS) were developed. Salient features of the proposed algorithms are: (i) Connectivity is defined as the ability to support end-to-end communication rates at a minimum prescribed level and not as a topological property of a graph. (ii) Uncertainty in the estimation of channel strength is incorporated into a statistical characterization of connectivity. (iii) The algorithms exploit spatial diversity to guarantee reliable end-to-end communication despite uncertain properties of point-to-point links.

The proposed algorithms were tested in an event-based simulator that uses an accurate data-driven model of radio communications to model both the structure of code running independently on multiple robots as well as the transmission of messages via a real radio. Simulation results corroborated that rates of end-to-end flows are maintained at target levels despite variations in rates of individual links.

\section{REFERENCES}

[1] H. Ando, Y. Oasa, I. Suzuki, and M. Yamashita, "Distributed memoryless point convergence algorithm for mobile robots with limited visibility,"
IEEE Transactions on Robotics and Automation, vol. 15, no. 5, pp. 818828, October 1999.

[2] Y. Kim and M. Mesbahi, "On maximizing the second smallest eigenvalue of a state-dependent graph laplacian," IEEE Transactions on Automatic Control, vol. 51, no. 1, pp. 116-120, January 2006.

[3] M. C. DeGennaro and A. Jadbabaie, "Decentralized control of connectivity for multi-agent systems," in Proc. 45th IEEE Conference on Decision and Control, San Diego, CA, December 2006, pp. 3628-3633.

[4] M. Ji and M. Egerstedt, "Coordination control of multi-agent systems while preserving connectedness," IEEE Transactions on Robotics, vol. 23, no. 4, pp. 693-703, August 2007.

[5] Y. Mostofi, "Communication-aware motion planning in fading environments," in Proc. Intnl. Conf. on Robotics and Automation, 2008, pp. 31693174.

[6] G. Notarstefano, K. Savla, F. Bullo, and A. Jadbabaie, "Maintaining limited-range connectivity among second-order agents," in Proc. 2006 American Control Conference, Minneapolis, MN, June 2006, pp. 2124 2129.

[7] M. M. Zavlanos and G. J. Pappas, "Potential fields for maintaining connectivity of mobile networks," IEEE Transactions on Robotics, vol. 23, no. 4, pp. 812-816, August 2007.

[8] M. Schuresko and J. Cortes, "Distributed motion constraints for algebraic connectivity of robotic networks," Journal of Intelligent and Robotic Systems, vol. 56, no. 1-2, pp. 99-126, September 2009.

[9] D. P. Spanos and R. M. Murray, "Motion planning with wireless network constraints," in Proc. 2005 American Control Conference, Portland, OR, June 2005, pp. 87-92.

[10] E. Stump, A. Jadbabaie, and V. Kumar, "Connectivity management in mobile robot teams," in Proc. IEEE International Conference on Robotics and Automation, Pasadena, CA, May 2008, pp. 1525-1530.

[11] I. Stojmenovic, A. Nayak, and J. Kuruvila, "Design guidelines for routing protocols in ad hoc and sensor networks with a realistic physical layer," IEEE Communications Magazine, vol. 43, pp. 101-106, March 2005.

[12] A. Neskovic, N. Neskovic, and G. Paunovic, "Modern approaches in modeling of mobile radio systems propagation environment," IEEE Communications Surveys, vol. 3, no. 3, pp. 1-12, 2000.

[13] H. Lundgren, E. Nordstrom, and C. Tschudin, "The gray zone problem in ieee $802.11 \mathrm{~b}$ based ad hoc networks," ACM SIGMOBILE Mobile Computing and Communications Review, vol. 6, no. 3, pp. 104-105, July 2002.

[14] J. Fink, N. Michael, A. Kushleyev, and V. Kumar, "Experimental characterization of radio signal propagation in indoor environments with application to estimation and control," St. Louis, MO, Oct. 2009.

[15] D. DeCouto, D. Aguayo, J. Bicket, and R. Morris, "A high-throughput path metric for multihop wireless routing," in Proc. ofInternational ACM Conference on Mobile Computing and Networking, San Diego, CA, September 2006, pp. 134-146.

[16] A. Ribeiro, Z.-Q. Luo, N. D. Sidiropoulos, and G. B. Giannakis, "Modelling and optimization of stochastic routing for wireless multihop networks," in Proc. 26th Annual Joint Conference of the IEEE Computer and Communications Societies (INFOCOM), Anchorage, Alaska, May 2007, pp. $1748-1756$.

[17] Y. Mostofi, A. Gonzalez-Ruiz, A. Gaffarkhah, and L. Ding, "Characterization and modeling of wireless channels for networked robotic and control systems - a comprehensive overview," in Proc. IEEE/RSJ Intnl. Conf. on Intelligent Robots and Systems, 2009, pp. 4849-4854.

[18] A. Ribeiro and G. Giannakis, "Separation theorems of wireless networking," IEEE Transactions on Information Theory, January 2008, (submitted).

[19] Y. Wu, A. Ribeiro, and G. B. Giannakis, "Robust routing in wireless multi-hop networks," in Proc. Conf. on Info. Sciences and Systems. Johns Hopkins Univ., Mar. 14-16, 2007, pp. 637-642.

[20] "CGAL, Computational Geometry Algorithms Library," http://www.cgal.org.

[21] R. Shorey, A. Ananda, M. C. Chan, and W. T. Ooi, Mobile, Wireless and Sensor Networks: Technology, Applications and Future Directions. Wiley, John \& Sons, Inc., 2006.

[22] K. T. Le, "Ieee 802.15.4 and zigbee compliant radio transceiver design,' http://www.hometoys.com/htinews/feb05/articles/chipcon/zigbee.htm, 2005.

[23] M. Quigley, K. Conley, B. Gerkey, J. Faust, T. Foote, J. Leibs, R. Wheeler, and $\mathrm{A}$. Y. Ng, "Ros: an open-source robot operating system," in ICRA Workshop on Open Source Software, 2009. 